\title{
Erratum to: Continuity of the Maximum-Entropy Inference
}

\section{Stephan Weis}

Max-Planck-Institute for Mathematics in the Sciences, Leipzig, Germany. E-mail: maths@weis-stephan.de

Received: 1 July 2014 / Accepted: 1 July 2014

Published online: 29 July 2014 - (C) Springer-Verlag Berlin Heidelberg 2014

Commun. Math. Phys. 330, 1263-1292 (2014)

In the original publication of the article, the author's name is incorrect. The correct name is Stephan Weis.

Communicated by A. Winter 